\title{
Percepción de los pacientes con insuficiencia renal crónica sobre los cuidados proporcionados por el personal de enfermería del centro de hemodiálisis Dialvida Ambato
}

\section{Perception of patients with chronic renal insufficiency about the care provided by the nursing staff of the hemodialysis center Dialvida Ambato}

\author{
Rosa Mercedes Castillo Siguencia ${ }^{1}$, Mishel Abigail Guerra Castillo ${ }^{1}$, Edison Gustavo Moyano Brito ${ }^{2}$, Nube \\ Johanna Pacurucu Avila ${ }^{2}$ \\ ${ }^{1}$ Universidad Técnica de Ambato, Ecuador \\ 2 Universidad Católica de Cuenca, Ecuador \\ *mguerra4913@uta.edu.ec
}

DOI: https://doi.org/10.26871/killkanasalud.v4i4.754

\begin{abstract}
Resumen
Introducción: La percepción de los pacientes con Insuficiencia Renal Crónica es muy importante para el mejoramiento en el desempeño del personal de Enfermería en las unidades de Hemodiálisis. Objetivo: El objetivo fue describir la percepción de los pacientes con insuficiencia renal crónica sobre los cuidados proporcionados por el personal de enfermería del Centro de Hemodiálisis Dialvida Ambato. Métodos: Se realizó un estudio de tipo cualitativo, descriptivo, transversal, de campo y prospectivo para conocer las ideas, pensamientos y opiniones de pacientes con insuficiencia renal crónica que se realizan hemodiálisis sobre el cuidado de enfermería. Por efecto de la misma, la técnica de estudio que se ejecutó fue la entrevista semiestructurada a un total de 14 pacientes cumpliendo el criterio de saturación de información. Resultados: Los resultados en la siguiente investigación fueron de tipo fenomenológico, conceptual y descriptivo de la percepción frente al cuidado de enfermería, cuidados humanizado e influencia del proceso de la enfermedad en la vida cotidiana. Conclusiones: Las conclusiones que se obtuvieron de la investigación fueron positivas y de satisfacción respecto al cuidado de enfermería, cuidado humanizado, pero se hallaron respuestas negativas como la falta de aceptación y adaptación a su cambio en el estilo de vida de los pacientes.
\end{abstract}

Palabras clave: insuficiencia renal crónica, cuidado humanizado, cuidado de enfermería, hemodiálisis.

\begin{abstract}
Introduction: The perception of patients with Chronic Renal Insufficiency is very important for the improvement in the performance of the Nursing staff in the Hemodialysis units. Objective: The objective was to describe the perception of patients with chronic renal failure about the care provided by the nursing staff of the Dialvida Ambato Hemodialysis Center. Methods: A qualitative, descriptive, cross-sectional, field and prospective study was carried out to find out the ideas, thoughts and opinions of patients with chronic renal failure who undergo hemodialysis on nursing care. Due to its effect, the study technique that was carried out was the semi-structured interview with a total of 14 patients, fulfilling the information saturation criterion. Results: The results in the following research were phenomenological, conceptual and descriptive of the perception of nursing care, humanized care and the influence of the disease process in daily life. Conclusions: The conclusions that were obtained from the research were positive and of satisfaction regarding the nursing care, humanized care, but negative responses were found such as the lack of acceptance and adaptation to their change in the patients' lifestyle.
\end{abstract}

Keywords: chronic kidney failure, humanized care, nursing care, hemodialysis.

\section{Introducción}

El cuidado de enfermería son procedimientos y técnicas que deben ser realizadas y cumplidas a cabalidad, pero además no se debe descuidar el cuidado humanizado del paciente, debido a que es una parte importante en su proceso de recuperación de la enfermedad. Por otra parte, 
se considera que la insuficiencia renal crónica ha causado un gran impacto a cientos de personas no solamente a nivel mundial sino también a nivel nacional como es en el Ecuador debido a que es una situación clínica en el que se encuentra presente el deterioro progresivo y terminal del funcionamiento de los riñones; por consiguiente, el papel de los profesionales de enfermería debe ser especializado y humanista en la atención a pacientes con insuficiencia renal crónica y que reciben diferentes tipos de tratamientos como pueden ser la hemodiálisis, diálisis peritoneal, reemplazo renal y entre otros.

Se puede señalar que los retos para el personal de enfermería respecto al cuidado directo de pacientes con distintos tipos de enfermedades crónicas degenerativas cada vez son más grandes. Según datos de la Organización Mundial de la Salud, entre estas enfermedades crónico degenerativas se encuentra la insuficiencia renal crónica, que es una enfermedad progresiva que no se propaga fácilmente y está muy relacionada con otras enfermedades como la enfermedad cardiovascular y la diabetes. ${ }^{1}$ Cuando se trata de esta enfermedad, afecta aproximadamente al $10 \%$ de la población mundial. Se puede prevenir pero no curar. Suele ser progresivo, silencioso, y los síntomas no aparecen hasta tarde, como soluciones (trasplante preventivo, diálisis (tratamiento entre domicilio y hospital), hemodiálisis diaria corta, hemodiálisis nocturna, diálisis peritoneal, diálisis diferida y cuidados paliativos) ya son muy invasivos y costosos. Muchos países carecen de recursos suficientes para comprar el equipo necesario o cubrir para todas las personas que necesitan estos tratamientos, el número de expertos disponibles tampoco es suficiente. ${ }^{2,3}$

De igual forma, la alta incidencia de enfermedad renal crónica ha causado gran preocupación a nivel mundial y en América Latina, por lo que la Sociedad Latinoamericana de Nefrología e Hipertensión (SLANH) afirmó que un promedio de 613 pacientes por millón de habitantes podrían utilizarlo en 2011. ${ }^{2}$ Considerando que Ecuador tiene 16 '278,844 residentes (fuente INEC), se estima que para el 2015, habrá 11,460 pacientes con insuficiencia renal. ${ }^{4}$ Por tanto, desde un punto de vista epidemiológico, es obvio que las cifras antes mencionadas indican que la prevalencia de la enfermedad es alta, lo que tiene un gran impacto en la sociedad.

Al mismo tiempo, es cierto que la profesión de enfermería se centra en el cuidado de la salud y la vida, por lo que el esfuerzo y el trabajo de las personas por los cuidadores, las personas, las familias y las comunidades se ven comprometidos. ${ }^{5}$

Es por ello, la importancia de los cuidados de enfermería en pacientes con insuficiencia renal crónica ya que existe un aumento de pacientes con este diagnóstico y que requieren cuidados asistenciales y especializados del personal de enfermería durante las sesiones de hemodiálisis, "la enfermera tiene la responsabilidad de garantizar que se logre el flujo sanguíneo prescrito", 6 además el personal debe cumplir con el rol de educación dirigida hacia el para el cuidado del paciente en casa por sus familiares o a través de autocuidado, para garantizar una adecuada recuperación y mantenimiento de la calidad de vida.

En este sentido, es comprensible que el rol de los profesionales de enfermería en el departamento de hemodiálisis se complique principalmente por los múltiples roles que deben asumir. Tecnólogos de enfermería, personal de enfermería profesional, educadores, facilitadores y consejeros emocionales. $\mathrm{Su}$ responsabilidad es integrar estos roles para establecer una relación terapéutica especial con cada paciente, lo cual es una tarea compleja. ${ }^{7}$

Ante esta situación el desarrollo de la investigación es importante pues tiene como objetivo describir la percepción de los pacientes con insuficiencia renal crónica sobre los cuidados proporcionados por el personal de enfermería del Centro de Hemodiálisis Dialvida Ambato.

\section{Materiales y métodos}

La investigación fue de tipo cualitativa, descriptiva y de campo pues la aplicación y recolección de datos a través de una entrevista se realizó en el Centro de Hemodiálisis Dialvida Ambato. ${ }^{8-10}$ Además, la investigación fue de corte trasversal y tuvo un enfoque prospectivo. ${ }^{11,12}$ La población objeto de estudio fueron los pacientes con diagnóstico de insuficiencia renal crónica que reciban tratamiento y atención de enfermería en hemodiálisis por más 1 año y de rango de edad de 30-70 años.

Este estudio, por ser cualitativo, no se estipula una muestra ni un muestreo, pues se tomó en cuenta la saturación de la información. La representatividad de estas muestras no radica en la cantidad de las mismas, sino en las posibles configuraciones subjetivas (valores-creenciasmotivaciones). ${ }^{13}$ Se logró la saturación de la información en 14 participantes de la investigación. Finalmente, como criterios de exclusión fueron los pacientes que reciban tratamiento de diálisis peritoneal, pacientes pediátricos y con patologías agregadas que limite dar información.

Previa a la aplicación de las entrevistas, se tomó en cuenta la variedad de pacientes por horarios y días de tratamiento. Además, se cumplieron con los protocolos de bioseguridad para el ingreso a la institución. Se explicó en términos claros y sencillos sobre los objetivos y el consentimiento informado. Posterior a firmar el consentimiento informado, se le informó y se le guía al lugar donde se realizó la entrevista. Las entrevistas realizadas fueron grabadas con la finalidad de mantener fielmente las respuestas de los participantes de la investigación.

Para realizar el análisis y procesamiento de datos fue necesario, categorizar, reducir, sintetizar, clarificar, comparar y sintetizar la información con el fin de contar con una visión completa de la realidad del objeto de estudio. ${ }^{14}$ En relación a lo anteriormente mencionado se empleó la teoría de codificación de Yuni y Urbano. Mientras que para la categorización y subcategorización se utilizó como referencia la teoría de Baéz y Tudela. ${ }^{15}$ 


\section{Resultados}

Después de la codificación de la información hallada en las entrevistas, se procedió a la categorización y sub categorización, con la finalidad de organizar la información obtenida para su posterior análisis.

Tabla 1: Categorización, subcategorización y codificación de resultados

\begin{tabular}{|c|c|c|c|}
\hline Tema central & Categoría & Subcategoría & Codificación \\
\hline \multirow[t]{12}{*}{$\begin{array}{l}\text { Percepción de los pacientes con insuficiencia renal crónica } \\
\text { sobre los cuidados proporcionados por el personal de enfer- } \\
\text { mería del centro de hemodiálisis Dialvida Ambato }\end{array}$} & \multirow{4}{*}{ Cuidado } & Autocuidado & $\begin{array}{l}\text { Estilo de vida } \\
\text { Alimentación } \\
\text { Actividad física } \\
\text { Higiene personal } \\
\text { Prevención de accidentes } \\
\text { Diagnóstico }\end{array}$ \\
\hline & & Asistencia sanitaria & $\begin{array}{l}\text { Asistencia médica } \\
\text { Sistema de salud } \\
\text { Tratamiento } \\
\text { Equipo multidisciplinario } \\
\text { Cuidado asistencial } \\
\text { Cuidado integral } \\
\text { Prescripción } \\
\text { Prevención de complicaciones }\end{array}$ \\
\hline & & Valores & $\begin{array}{l}\text { Hermandad } \\
\text { Solidaridad } \\
\text { Apoyo } \\
\text { Compañerismo }\end{array}$ \\
\hline & & Red de apoyo & $\begin{array}{l}\text { Familia } \\
\text { Hogar }\end{array}$ \\
\hline & \multirow[t]{3}{*}{$\begin{array}{c}\text { Cuidado } \\
\text { Humanizado }\end{array}$} & Valores & $\begin{array}{l}\text { Empatía } \\
\text { Solidaridad } \\
\text { Caritativo } \\
\text { Humanidad } \\
\text { Amabilidad } \\
\text { Compañerismo }\end{array}$ \\
\hline & & Sentimientos & Preocupación \\
\hline & & Cuidado asistencial & $\begin{array}{l}\text { Monitorización de signos vitales } \\
\text { Tratamiento }\end{array}$ \\
\hline & \multirow[t]{5}{*}{$\begin{array}{l}\text { Cuidado de } \\
\text { Enfermería }\end{array}$} & Cuidado asistencial & $\begin{array}{l}\text { Gestión de sistema de salud } \\
\text { Ayuda } \\
\text { Cuidado integral } \\
\text { Educación } \\
\text { Tratamiento } \\
\text { Sistema de salud } \\
\text { Control de signos vitales } \\
\text { Medidas de bioseguridad } \\
\text { Desinfección del entorno } \\
\text { Informe de enfermería }\end{array}$ \\
\hline & & $\begin{array}{c}\text { Cuidado } \\
\text { especializado }\end{array}$ & $\begin{array}{l}\text { Conocimientos previos en dializadores } \\
\text { Capacitación del uso de dializadoras } \\
\text { Practica previa en dializadores } \\
\text { Procedimiento invasivo } \\
\text { Equipo multidisciplinario } \\
\text { Manejo de complicaciones } \\
\text { Prevención de complicaciones } \\
\text { Complicaciones } \\
\text { Condición clínica } \\
\text { Cuidado integral }\end{array}$ \\
\hline & & $\begin{array}{l}\text { Personal de } \\
\text { enfermería }\end{array}$ & $\begin{array}{l}\text { Virtud } \\
\text { Amabilidad } \\
\text { Pulcritud } \\
\text { Cortesía } \\
\text { Actitud positiva }\end{array}$ \\
\hline & & $\begin{array}{c}\text { Relación } \\
\text { paciente-enfermera }\end{array}$ & $\begin{array}{l}\text { Falta de comunicación } \\
\text { Incumplimiento del tratamiento } \\
\text { Desinformación } \\
\text { Cumplimiento en el tratamiento } \\
\text { Hecho percibido }\end{array}$ \\
\hline & & $\begin{array}{l}\text { Expectativas y } \\
\text { experiencias }\end{array}$ & $\begin{array}{l}\text { Satisfacción } \\
\text { Experiencias positivas } \\
\text { Experiencias negativas }\end{array}$ \\
\hline
\end{tabular}


Tabla 2: Categorización, subcategorización y codificación de resultados...(continuación)

\begin{tabular}{|c|c|c|c|}
\hline Tema central & Categoría & Subcategoría & Codificación \\
\hline & \multirow[t]{4}{*}{$\begin{array}{l}\text { Influencia del } \\
\text { diagnóstico y } \\
\text { tratamiento en la } \\
\text { vida cotidiana }\end{array}$} & Conocimiento & $\begin{array}{l}\text { Antecedente patológico personal } \\
\text { Antecedentes patológicos familiares } \\
\text { Desinformación } \\
\text { Síntomas } \\
\text { Complicaciones post-diálisis } \\
\text { Alteración mental } \\
\text { Diagnóstico }\end{array}$ \\
\hline & & $\begin{array}{l}\text { Emociones y } \\
\text { sentimientos }\end{array}$ & $\begin{array}{l}\text { Incertidumbre } \\
\text { Tristeza } \\
\text { Sentimientos positivos } \\
\text { Temor } \\
\text { Desesperanza }\end{array}$ \\
\hline & & Autocuidado & $\begin{array}{l}\text { Tratamiento } \\
\text { Desatención } \\
\text { Complicación }\end{array}$ \\
\hline & & Nueva perspectiva & $\begin{array}{l}\text { Hechos percibidos } \\
\text { Negación } \\
\text { Personal médico } \\
\text { Hogar } \\
\text { Trabajo } \\
\text { Familia } \\
\text { Aceptación } \\
\text { Adaptación } \\
\text { Convivencia } \\
\text { Apoyo } \\
\text { Modificación del estilo de vida } \\
\text { Motivación }\end{array}$ \\
\hline
\end{tabular}

Fuente: Entrevista a los pacientes con insuficiencia renal crónica sobre los cuidados proporcionados por el personal de enfermería del Centro De Hemodiálisis Dialvida Ambato

\section{Aproximación teórica}

4.1 Definición de los cuidados proporcionados por el personal de enfermería según la percepción del paciente con insuficiencia renal crónica

El cuidado de enfermería a pacientes con insuficiencia renal crónica es el conjunto de actividades y procedimientos técnicos especializados ejecutados con responsabilidad, conocimientos previos en el manejo de dializadoras, estos deben ser planificados de acuerdo a la característica y valoración clínica de cada paciente con la finalidad de que durante el proceso de hemodiálisis se cumpla la prescripción médica, además de la prevención y manejo de complicaciones de manera oportuna.

Por esta razón el personal de enfermería cumple roles y funciones asistenciales, administrativas, investigación y educación. Siendo necesaria la gestión de salud en cumplimiento de la necesidad y requerimiento de cada paciente ya que presentan otras patologías asociadas a la insuficiencia renal crónica y deben ser atendidos en distintas casas de salud en caso de ser requerido la asistencia médica de otras especialidades. De esta manera lograr la satisfacción de las necesidades de los pacientes y cumplir con todas las expectativas para mejorar su calidad de vida.

\subsection{Descripción de la definición de cuidado humanizado desde la perspectiva del paciente con insuficiencia renal crónica}

El cuidado humanizado desde la perspectiva de los pacientes se basa primordialmente en la humanidad, la empatía, solidaridad y la preocupación por el otro. Los pacientes no ingresan solos a una sala de diálisis, se encuentran en compañía de muchas otras personas que se van a someter a un mismo proceso, pero cada individuo es un mundo diferente y requiere de distintos cuidados a más de los procesos técnicos de la hemodiálisis. Muchos de ellos aplican el compañerismo ya que la convivencia y la asistencia al cumplimento de la hemodiálisis y la aceptación del mismo es difícil y diferente para cada persona.

\section{Discusión}

\subsection{El cuidado}

Para definir el cuidado es necesario conocer que esta palabra etimológicamente proviene del término "cuidare/curare". ${ }^{16}$ Del mismo modo se entiende que desde el inicio de la vida debe estar presente el cuidado como una estrategia de protección de riesgos posibles para la salud e integridad, que es aprendida desde temprana edad. ${ }^{17} \mathrm{En}$ este contexto se puede correlacionar la información que se recopilo de las entrevistas en el que "Cuidado, tener mucha disciplina, en cuidarse uno con los alimentos, saberse cuidar bastante y no desmandarse que más le podría decir, más que todo por la diálisis que se hace, toca hacerse terapia, hacerse deporte es lo que mantiene más al paciente..." (Seudónimo: Jackson, S).

De manera similar, la palabra çuidar"puede conceptualizarse como un proceso dinámico e interactivo. Se compone de las siguientes categorías: inclusión familiar, interacción interpersonal y atención distintiva (centrada en las personas). En los códigos involucrados en estas categorías, hay relaciones humanas dentro del significado, estas relaciones 
humanas encarnan el intercambio de acciones o ideas para fines específicos, e incluyen el respeto y la individualización en entidades con características propias y específicas. Al mismo tiempo, en esta dinámica, se ha comprobado el papel fundamental de la comunicación y el interés por los demás, lo cual es una promesa de compasión de manera sincera ante las peticiones especiales de los individuos que viajan cuando lo necesitan. ${ }^{18}$

\subsection{Cuidado Humanizado}

Watson, autor de The Theory of Humanistic Care, cree que dada la estructura, los nuevos modelos y los cambios tecnológicos pueden conducir a la deshumanización de la atención de la salud, los profesionales de enfermería deben salvar los aspectos humanos, espirituales y sobrehumanos. ${ }^{19}$ Por tanto, el cuidado debe brindarse de manera personalizada, es decir, al realizar el cuidado, siempre debemos considerar a las personas que tenemos frente a nosotros, porque todos somos maravillosos, únicos e irrepetibles. ${ }^{20}$ Los pacientes son el principal motivo por el que debemos mantener un cuidado humano . ${ }^{\mathrm{A}} \mathrm{quí}$... nos pueden ayudar en todos los aspectos, nos dan la mano en todos los aspectos, pero también hay que darles a algunos compañeros que sufren mucho por la diálisis ..."(Alias: Peterson (W) Sin embargo, en el estudio de Herrera et al., Los resultados mostraron un alto nivel de comprensión del cuidado humanizado. El puntaje más bajo es promover la fe y la esperanza $(\mathrm{M}=3.56 ; \mathrm{SD}=0.19)$ para promover y aceptar la expresión de sentimientos positivos y negativos $(\mathrm{M}=2.07 ; \mathrm{SD}=0.25),{ }^{21} \mathrm{y}$ en esta encuesta, describimos . ${ }^{A}$ tención interpersonal, porque nos cuidamos unos a otros como pacientes, por eso nos preocupa que ese tipo de gente no venga, sentimos que nos ayudamos unos a otros ... "(seudónimo: Tesla, N)

\subsection{Cuidado de enfermería a pacientes con insuficiencia renal crónica de un centro de hemodiálisis}

Para lograr un adecuado cuidado a pacientes con insuficiencia renal crónica con tratamiento de hemodiálisis, es necesario personal apto ya capacitado para desempeñar las distintas funciones por ello entre las respuestas se encontró “... deben ser unas personas capacitadas especialmente en hemodiálisis, deben capacitarles antes de mandarles para acá, a veces no sacan bien las agujas, es necesario personas capacitadas y con mucha paciencia..." (Seudónimo: Orem, D)

Así mismo, el cuidado de enfermería comprende aspectos afectivos, relativos a la actitud, conocimiento, valores, habilidades, así como elementos técnicos que no pueden ser separados para otorgar cuidados, incluyen también la identificación del significado del cuidado para quien lo otorga y para quien lo recibe, la intención y la meta que se persigue. $^{22}$

Sin embargo, en la investigación de López et al en el año 2018 en Colombia obtuvieron los resultados fueron que entre el $60 \%$ y $85 \%$ de la población evaluaba de manera positiva los cuidados proporcionados por el personal de enfermería. ${ }^{23}$ En relación al presente estudio se encontraron percepciones positivas como la siguiente respuesta: "El personal de enfermería muy bueno, nos atiende mucho, nos cuidan bastante, se preocupan por nosotros, cuando a veces necesitamos que nos ayuden con los turnos para el hospital nos dan, también cuando tenemos muchos problemas nos guían.” (Nightingale, F).

Por otro lado, Vásquez et al en el año 2019 confirma que acciones como la provisión de información, la educación, el entrenamiento y seguimiento son claves para lograr un éxito en la práctica de cuidado. ${ }^{24}$ En contexto con lo anteriormente mencionado, los cuidados de enfermería van de la mano con la información, preparación y capacitación previa, la educación y el seguimiento

\subsection{Influencia del diagnóstico y tratamiento en la vida cotidiana}

Está claro que exponer sobre el impacto que este tipo tratamiento ejerce sobre un paciente de hemodiálisis, básicamente por la exposición a distintas fuentes de ansiedad, tales como: dependencia a una máquina y al equipo médico, restricción dietética, tratamiento multifarmacológico, dificultad para replantear y planificar su futuro, entre otras. ${ }^{25}$ Es por ello que entre las respuestas se halla la negación y la aceptación de la enfermedad como se da en esta respuesta "Me ha afectado, hasta ahora no lo acepto, aceptarlo sería venir acá como si nada y hacer la vida normal como si fuera parte de mi vida, pero no, no puedo. Me ha afectado en todo sentido, no sé cuándo lo aceptare" (Seudónimo: Evans, C).

Por tanto, la respuesta y la información obtenida están directamente relacionadas con el estudio realizado por $\mathrm{Pa}-$ bón et al. En 2015. Los resultados del estudio indican que la dimensión de la calidad de vida más afectada es una fisioterapia para la insuficiencia renal, que afecta la vida. de pacientes El mayor factor de calidad es la hemodiálisis. ${ }^{26}$

Cabe destacar que para una persona con IRC someterse a hemodiálisis puede ser una realidad impuesta, muchas veces sin tener noción de lo que el tratamiento implica en su vida. Es aquí en donde las personas experimentan la aceptación o no de esta nueva realidad, en donde las personas pueden esforzarse por mantener la vida que mantenían antes del tratamiento o bien, pueden refugiarse en la nueva vida que conlleva, dejando trabajos, actividades de gusto o entorno social. ${ }^{27}$

En este contexto, las respuestas en relación al cambio y la influencia del diagnóstico en la vida cotidiana de las personas se han visto de la siguiente manera: "Esta enfermedad al menos para mí personalmente es un cambio de vida total, es una enfermedad silenciosa..." (Seudónimo: Jackson, S). La aceptación y el aprendizaje es algo muy distinto en cada persona siendo esencial el apoyo familiar.

Se puede observar que en el estudio de 2018 de Gómez et al., El 86,7 \% de las personas cree que el papel de la familia en todos los procesos crónicos de los pacientes es crucial, 
porque exhibe cambios y por tanto se adapta al eje de su proceso y procesamiento. ${ }^{28}$

Por lo tanto, la felicidad de todos depende de sus propios valores, creencias, antecedentes culturales e historia de vida personal. Por lo tanto, las personas con afecciones clínicas similares experimentarán afecciones diferentes. ${ }^{29}$ La calidad de vida incluye 4 aspectos básicos: salud física (autonomía y capacidad física), malestar físico (síntomas provocados por enfermedad y tratamiento), estado mental (emoción, ansiedad y depresión) y problemas de relación social (familiar y profesional), lo que significa que Es necesario considerar el malestar físico independientemente del dominio físico. ${ }^{30}$ Por lo que, al hablar de la vida cotidiana de una persona con IRC es necesario conocer que existe un impacto grande en los dominios antes mencionados que afectan a cada persona desde el diagnóstico y consecuentemente en el tratamiento, como se menciona en la respuesta:

"Al comienzo fue bien duro que tenía ganas hasta de quitarme la vida, porque fue un golpe bien duro, tengo un pequeñito de 7 años eso cambio totalmente mi vida, dio un giro bastante fuerte en mi vida pero gracias a las personas al psicólogo a la trabajadora social y a todos en si aquí, me han ayudado a tomar mi enfermedad como que día a día que va pasando, tomando en cuenta que es una enfermedad que va a estar conmigo hasta cuando Dios nos preste la vida y en mi casa igual mis padres y más que todo verle a mi hijo me ha dado fuerzas para seguir luchando y poder enfrentar esta enfermedad que no es nada fácil pero ahí le damos" (Seudónimo: Stark, T)

\section{Conclusiones}

Se describieron los cuidados proporcionados por el personal de enfermería según la percepción del paciente con insuficiencia renal crónica del Centro de Hemodiálisis Dialvida Ambato, los cuales fueron obtenidos a través de la codificación, categorización y subcategorización de las respuestas. Los cuidados fueron definidos exitosamente pues se abarca no solo el cuidado asistencial sino también el procedimiento técnico especializado que realiza el personal de enfermería y los retos a los cuales se enfrenta como es la prevención y tratamiento oportuno de complicaciones

En cuanto al cuidado de enfermería se recomienda realizar un estudio longitudinal con la finalidad de que se conozca a profundidad todos los procedimientos de enfermería en el Centro de Hemodiálisis y el nivel de cumplimiento de cada uno con relación a los objetivos y expectativas de cada paciente.

\section{Fuente de Financiamiento}

Este estudio es autofinanciado.

\section{Conflicto de Intereses}

No existen conflictos personales, profesionales, financieroso de otro tipo.

\section{Consentimiento Informado}

Los autores cuentan con el consentimiento informado de los pacientes para la investigación, la publicación del caso y sus imágenes.

\section{Referencias Bibliográficas}

1. Lopera-Medina MM. La enfermedad renal crónica en Colombia: Necesidades en salud y respuesta del Sistema General de Seguridad Social en Salud. Rev Gerenc y Polit Salud. 2016 Jan 1;15(30):212-33.

2. PAHO/WHO I PAHO and Latin American Society of Nephrology call for increased prevention and better access to treatment for kidney disease [Internet]. [cited 2021 Feb 20]. Available from: https://www.paho.org/hq/index.php?option=com content \&view $=$ article $\&$ id $=10542: 2015$-opsomssociedad-latinoamericana-nefrologia-enfermedadrenal-mejorar-tratamiento \&Itemid=1926\&lang=en

3. Red R. Manual de dialisis daugirdas 4a ed [Internet]. [cited 2021 Feb 20]. Available from: https://www.academia. edu/28497488/Manual_de_dialisis_daugirdas_4a_ed

4. Presentación Diálisis Criterios de Priorización y Planificación.pdf [Internet]. [citado 28 de octubre de 2020]. Disponible en: https://aplicaciones.msp.gob.ec/ salud/archivosdigitales/sigobito/tareas_seguimiento/ 1469/Presentaci\%C3\%B3n\%20Di\%C3\%A1lisis\% 20Criterios $\% 20 \mathrm{de} \% 20$ Priorizaci\%C3\%B3n\%20y\% 20Planificaci\%C3\%B3n.pdf

5. Lagoueyte Gómez María Isabel. El cuidado de enfermería a los grupos humanos. Rev. Univ. Ind. Santander. Salud [Internet]. 2015 Aug [citado 30 octubre de 2020]; 47( 2 ): 209-213. Disponible en : http://www.scielo.org.co/scielo.php?script=sci_arttext\& pid=S0121-08072015000200013\&lng=en.

6. Thomas N. Renal Nursing [Internet]. 4th ed. London: WILEY Blackwell; 2014 [citado 9 de Noviembre de 2020]. Disponible en: https://ebookcentral.proquest.com/ lib/uta-ebooks/reader.action?docID=1524294\&query= renal+nursing+

7. Partida K, Tejada L, Chavez Y, Hernandez O, Lozano M. El cuidado de enfermería a los enfermos renales en hemodiálisis: desafíos, dilemas y satisfacciones. Revista da Escola de Enfermagem da USP [Internet]. 2019 [citado 5 de Noviembre de 2020];:2. Disponible en : https://www.scielo.br/scielo.php?pid=S008062342019000100484\&script=sci_arttext

8. Sampieri, R. (2006). Enfoque cualitativo y cuantitativo, según Hernández Sampieri.. [online] Portafolio académico. Disponible en : https: //portaprodti.wordpress.com/enfoque-cualitativo-ycuantitativo-segun-hernandez-sampieri/ [Accessed 16 oct.-20].

9. Guadalupe, S., Castillo, R., Fernandez, M. and Herrera, J. (2017). Enfermeria Investiga. [Internet] Fcs.uta.edu.ec. Disponible en: https://fcs.uta.edu.ec/v3.2/investigacion/ 
libros \%20enfermeria/LIBRO\%20ENFERMER\%C3\% 8DA\%20INVESTIGATIVA.pdf [Accessed 16 oct.20].

10. Sanchez H, Reyes C, Mejia K. Manual de términos en investigación científica, tecnológica y humanística [Internet]. Lima: Universidad Ricardo Palma;21. 2018 [citado 20 de Octubre de 2020]. Disponible en : http://repositorio.urp.edu.pe/handle/URP/1480

11. Manterola C, Quiroz G, Salazar P, Garcia N. Metodología de los tipos y diseños de estudio más frecuentemente utilizados en investigación clínica. Revista Medica Clinica Las Condes [Internet]. 201922. [citado 8 de Octubre de 2020];(30):36-49. Disponible en: https://www.sciencedirect.com/science/article/pii/ S0716864019300057

12. Modelos De Estudios En Investigación Aplicada: Conceptos Y Criterios Para El Diseño. Med Segur Trab [Internet]. 2008 [cited 8 October 2020];(210):7-8. Disponible en: http://scielo.isciii.es/pdf/mesetra/v54n210/aula.pdf

13. Serbia J. Diseño, muestreo y análisis en la investigación 23 cualitativa. Hologramática [Internet]. 2007 [cited 25 November 2020];(7):123-133. Available from: http://dspace. utalca.cl/bitstream/1950/9421/1/Serbia_JM.pdf

14. Guerrero M. La investigación cualitativa. Revista de la Universidad Internacional del Ecuador [Internet]. 2016 [citado 25 de Noviembre de 2020];:1-9. Disponible en: https://revistas.uide.edu.ec/index.php/innova/ article/view/7/1442

15. Urbano P. Análsis de datos cualitativos. Revista Fedumar Pedagogía y Educación [Internet]. 2016 [citado 25 de Noviembre de 2020];(3):113-126. Disponible en: http://editorial.umariana.edu.co/revistas/index. php/fedumar/article/view/1122/1064

16. Alba Martín - 2015 - El concepto de cuidado a lo largo de la historia.pdf [Internet]. [citado 28 de noviembre de 25 . 2020]. Disponible en: https://rua.ua.es/dspace/bitstream/ 10045/46617/1/Cultura-Cuidados_41_12.pdf

17. Becerra A, Guarnizo M, Vargas L. Significado del cuidado antes de iniciar la formación profesional en Enfermería. Educación Médica Superior [Internet]. 2018 [Citado 2 de diciembre de 2020];32(3):133-146. Disponi- 26. ble en: http://scielo.sld.cu/scielo.php?script=sci_arttext\& pid=S0864-21412018000300011

18. Ramírez Elizondo N. Significado, relevancia y elementos de género asociados al cuidado: metasíntesis cualitativa. Index de Enfermería[Internet]. 2016 [Citado 28 de noviembre de 2020];25(1-2):33-7. Disponible en: http://scielo.isciii.es/scielo.php?script=sci_27. arttext\&pid=S1132-12962016000100008

19. Monje P, Miranda P, Oyarzün J, Seguel F, Flores E. Percepción de cuidado humanizado de enfermería desde la perspectiva de usuarios hospitalizados. Ciencia y Enfermeria [Internet]. 2018 [ci-28. tado 2 de diciembre de 2020];24(5):1-10. Disponible en: https://scielo.conicyt.cl/scielo.php?script=sci_ arttext\&pid=S0717-95532018000100205

20. Vanegas P. Cuidado humanizado: un desafío para el pro- fesional de enfermería. REVISALUD Unisucre [Internet]. 2015 [citado 30 de Noviembre de 2020];3(1):26-30. Disponible en: https://www.recia.edu.co/index.php/revisalud/ article/view/575/620

- Herrera V, Tandazo B, Idrovo M. Percepción de cuidado humanizado de enfermería en pacientes con insuficiencia renal crónica. Conecta Libertad [Internet]. 2020 [citado 4 de Noviembre de 2020];:1. Disponible en: http://revistaitsl.itslibertad.edu.ec/index.php/ITSL/ article/view/93

. Lascano M, Barrera E, Cedeño D, Hernandez N, Segovia $\mathrm{A}$, Jimenez $\mathrm{R}$ et al. Competencias del personal de enfermería en el paciente con hemodiálisis. Educación y Salud Boletín Científico Instituto de Ciencias de la Salud Universidad Autónoma del Estado de Hidalgo [Internet]. 2017 [citado 30 de Noviembre de 2020]; 6(11). Disponible en: https://repository.uaeh.edu.mx/revistas/index.php/ ICSA/article/view/2722/2746

Alfonso López-Soracipa V, Alexandra EspañaBarrios E, Elena Hernández-Garcés A. Estimación de calidad del cuidado de enfermería en pacientes sometidos a hemodiálisis. Revista Ciencia y Cuidado. 1 de enero de 2018;15:71. Disponible en: https://www.researchgate.net/publication/323827915_

Estimacion_de_calidad_del_cuidado_de_enfermeria_en_ pacientes_sometidos_a_hemodialisis

24. Vásquez C. Plan de cuidados de enfermería en pacientes insuficientes renales atendidos en medicina interna del hospital San Vicente de Paúl 2019 [Licenciatura]. Universidad Tecnica del Norte; 2019. Disponible en: http://repositorio.utn.edu.ec/bitstream/123456789/ 9851/2/06\%20ENF\%201141\%20TRABAJO\%20DE\% 20GRADO.pdf

5. Cevallos A, Anchundia A, Velez M, Cedeño C, Santana Z, Agul M. Calidad de vida en pacientes con tratamiento terapéutico de hemodiálisis. Dominio de las Ciencias [Internet]. 2019 [citado 30 de Noviembre de 2020];(1):3-31. Disponible en: https://dialnet.unirioja.es/servlet/articulo? codigo $=6869946$

6. Pabón-Varela Y, Paez-Hernandez KS, RodriguezDaza KD, Medina-Atencia E, López-Tavera M. Adult's life quality with chronic kidney disease, A bibliographic view. 2015;12:8. Disponible en: https://www.redalyc.org/pdf/5121/512156300010.pdf? fbclid=IwAR2BjpoIaFbaNQqH-5S0b9GRfAoQUWCKKmaicvLs-oBDYm64WfQVt4FqQY

27. Andrade M, Guerreiro D, Rozza S. Vida después de la hemodiálisis. Texto Contexto Enferm [Internet]. 2020 [citado 2 de Diciembre de 2020];(29):1-15. Disponible en: https://www.scielo.br/scielo.php?pid=S010407072020000600309\&script=sci_arttext\&tlng=es

. Martínez NG, Martínez NG, Sánchez F de JC, Caiza JNT. Intervención de enfermería en el estado emocional de pacientes hemodializados en el Hospital General Ambato. Enfermería Investiga: Investigación, Vinculación, Docencia y Gestión. 2018;3(3):142-8. Disponible en: https:// 
dialnet.unirioja.es/servlet/articulo?codigo $=6602842 \mathrm{P}$

29. Lleixà M, Casadó L, Andújar J, Solà Miravete E, Martínez E, Salvadó T. Valoración de la calidad de vida relacionada con la salud en la enfermedad renal crónica terminal mediante un cuestionario de resultados percibidos por los pacientes. Enfermería Nefrológica [Internet]. 2016 [citado 3 de Diciembre de 2020]; 19(4):331340. Disponible en: http://scielo.isciii.es/scielo.php?pid= S2254-28842016000400004\&script=sci_arttext\&tlng=pt

30. Krederdt S, Taboada D, Meléndez E. Calidad de vida percibida en pacientes con insuficiencia renal crónica terminal ambulatorios. Servicio nefrología hospital nacional Alberto Sabogal Sologuren -ESSALUD. Callao. Revista Cientifica de Enfermeria [Internet]. 2020 [citado 3 de Diciembre de 2020];(2):2-15. Disponible en: https://revista. cep.org.pe/index.php/RECIEN/article/view/26/25

Recibido: 9 de octubre de 2020

Aceptado: 25 de octubre de 2020

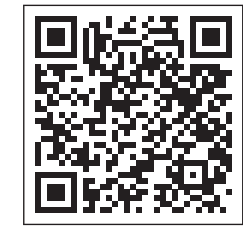

